www.jmscr.igmpublication.org

Impact Factor 3.79

Index Copernicus Value: 5.88

ISSN (e)-2347-176x ISSN (p) 2455-0450

crossref DOI:_http://dx.doi.org/10.18535/jmscr/v3i12.05

Journal Of Medical Science And Clinical Research

\title{
Childhood Blindness and Subnormal Vision- Evaluation and Management at AVBRH - A Rural Hospital Based Study
}

\author{
Authors \\ Dr Prachee Nagrale ${ }^{1}$, Dr. Mala Kamble ${ }^{2}$, Dr. Pradeep Sune ${ }^{3}$, Dr. Somya Dulani ${ }^{4}$ \\ ${ }^{1}$ Assistant Professor, Department of Ophthalmology, JNMC, Sawangi, Wardha \\ ${ }^{2}$ Professor, Department of Ophthalmology, JNMC, Sawangi, Wardha \\ ${ }^{3}$ Professor, Department of Ophthalmology, JNMC, Sawangi, Wardha \\ ${ }^{4}$ Professor, Department of Ophthalmology, JNMC, Sawangi, Wardha \\ Corresponding Author \\ Dr Prachee Nagrale
}

204, Penna Building, Mamata Hospital Campus, Giriprasad Nagar, Khammam, Telangana 507002 India

Email: pracheenagrale@yahoo.co.in, Mobile Number: 09959093035

\begin{abstract}
Vision is the most important special sense in human being. Normal vision is essential for normal physical, mental, psychological development and education childhood blindness refers to a group of disease and conditions occurring in childhood which if left untreated results in blindness or severe visual impairment later in life so this study is done to find out prevalence of blindness and subnormal vision in paediatric age group, to evaluate the main site of abnormality leading to visual loss and, to treat and evaluate the prognosis for these visually subnormal or blind children, and to visually rehabilitate children to best possible extent. Total 600 paediatric patients from age group 0-16 years attending eye OPD at AVBH, Sawangi(Meghe), Wardha with complaints of blindness or subnormal vision having visual acuity less than $6 / 18$ were included in this study. Thus we found the cases as contract (Congenital, Developmental, traumatic) 16\%, Refractive error 15.33\%,Congenital global anamolies (Microphthalmos, Anaphthalmos) 4.99\%,Ptosis 11.6\%,Corneal disorders (opacity, keratoconus) 10.48\%Posterior segment pathologies - 7.29\%,Amblyopia 13.33\%, Ocular trauma 3\%, Idiopathic Nystagmas 3.66\%, Xerophthalmia 4.16\%, Therefore Preventable causes of blindness can be tackled by vitamin A supplementation, rubella \& measles immunization. Treatable causes needs early detection and immediate treatment for improvement of ultimate visual prognosis in cases such as congenital cataract, uncorrected refractive errors, amblyopia, congenital glaucoma. Creating public awareness and screening programms in community and school is essential for early detection and management.
\end{abstract}

Key words: Childhood blindness, early detection, early treatment, awareness programms

\section{INTRODUCTION}

Childhood blindness refers to a group of diseases and conditions occurring in childhood, which, if left untreated, result in blindness or severe visual impairment which are likely to be untreatable later in life.
Due to enormous loss of Disability Adjusted Life Years (DALYs), childhood blindness is estimated to be the second leading cause of the burden due to blindness.

Without visual stimulus, the child's overall development suffers. 
Because of the country's size, the eye care sector in India faces several major challenges including lack of access for rural populations, unequal distribution of services, gross underutilization of available services, and insufficient facilities.

\section{Objectives of this study were}

To find out prevalence of blindness and subnormal vision in paediatric age group.To evaluate the main site of abnormality leading to visual loss.To find out the etiology of visual loss. To treat and evaluate the management and prognosis for these visually subnormal or blind children.To visually rehabilitate patients to the best possible extent.

WHO defines blindness as a corrected visual acuity in the better eye of less than $3 / 60$ and severe visual impairment as corrected visual acuity in the better eye of less than $6 / 60$ but equal to, or better than $3 / 60$.

Age group 0-16years were included in this study

Patients having visual acuity less than $6 / 18$ were included in this study

Thus patients more than 16 years of age and Patients having visual acuity more than $6 / 18$ were excluded
In this study total 600 paediatric patients from age group of 0-16years attending Eye OPD from the period of May 2008 to June 2010 complaining of blindness or subnormal vision, having visual acuity less than 6/18 were included in the study.

History in detail was taken, Visual Acuity Assessment was done, Ocular Examination using torch light and slit- lamp was perform, Fundus Examination using Direct and Indirect Ophthalmoscopy was done, Ocular Movements and Alignment was assessed, B-Scan wherever indicated was done.

Thus in our study, of the 600 children studied, we found Cataract (Congenital, Developmental, Traumatic) $\quad 16 \%$, Refractive Error 15.33\%,Congenital Globe Anomalies (Microphthalmos, Anophthalmos)4.99\%, Ptosis $11.6 \%$, Corneal Disorders (Opacity, Keratoconus) $10.48 \%$,Posterior Segment Pathologies (Macular DystrophyROP, Retinoblastoma, Toxoplasma Chorioretinitis Pathological Myopia, Traumatic R.D) $7.29 \%$, Amblyopia 13.33\%, Ocular Trauma $3 \%$,Idiopathic Nystagmus 3.66\%,Xerophthalmia $4.16 \%$.

Graph 1 shows no. of cases according to type of disease

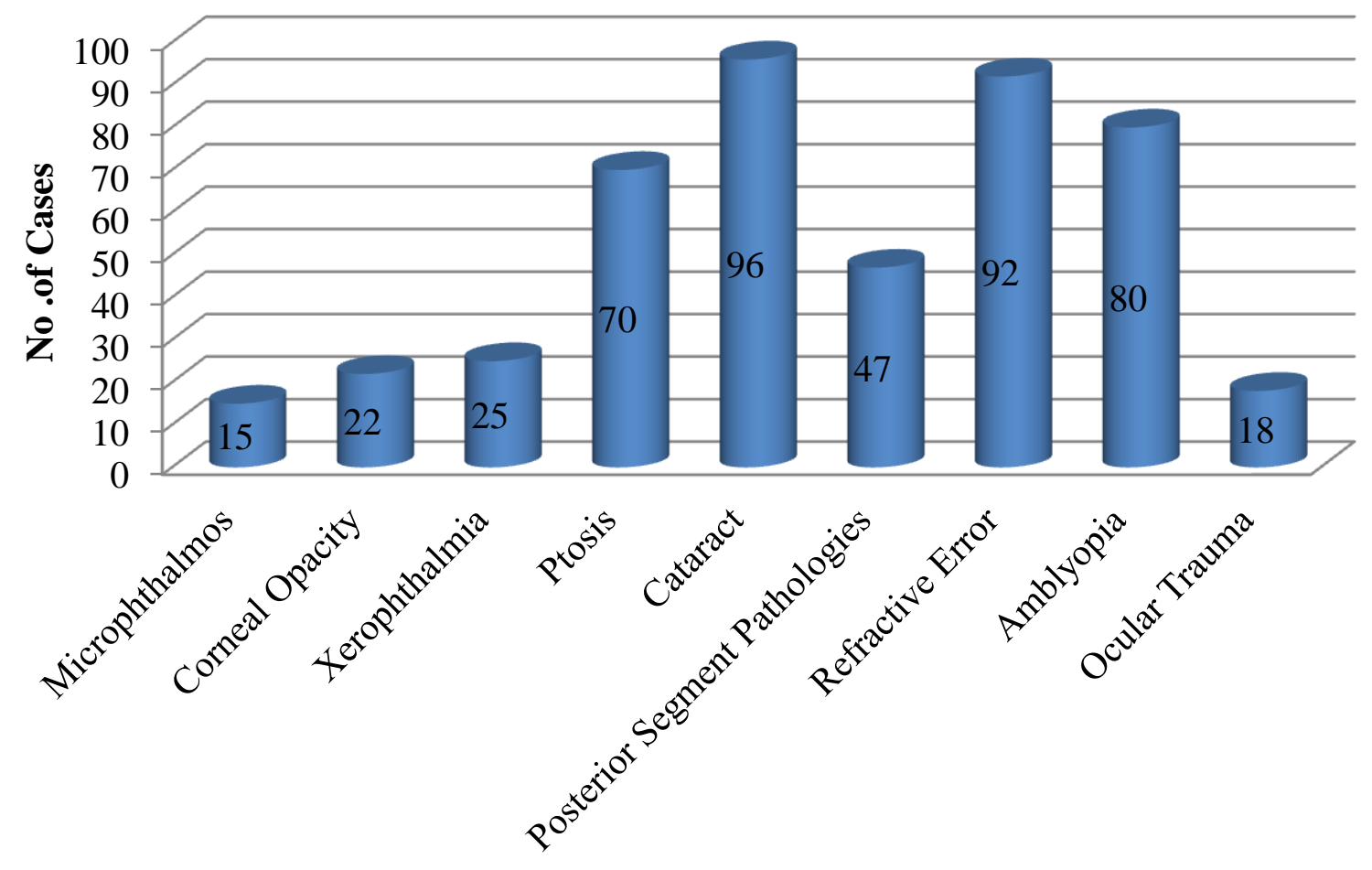




\section{JMSCR Vol||3||Issue||12||Page 8477-8489||December}

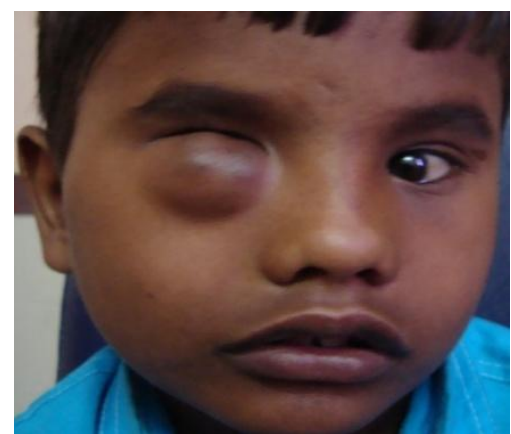

Anophthalmos with cyst 4 cases $(0.66 \%)$

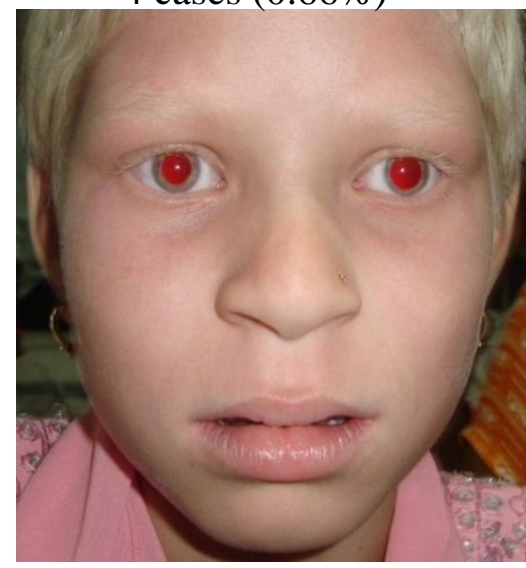

Albinism 4 cases $(0.66 \%)$

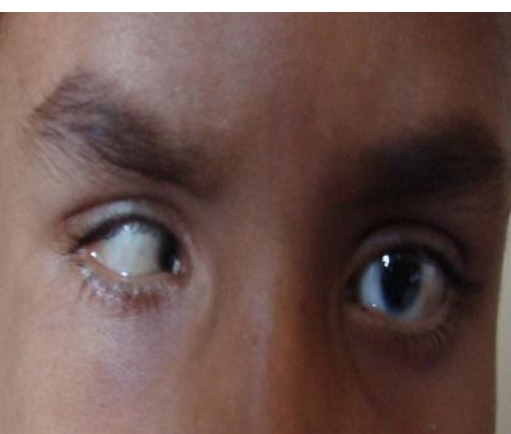

Microphthalmos with microcornea 15 cases $(2.5 \%)$

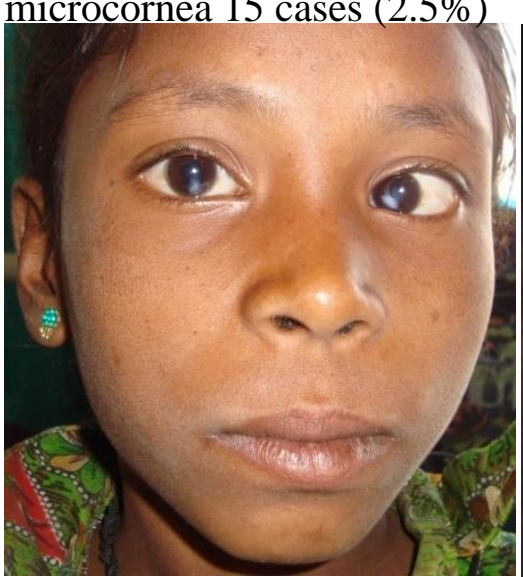

Bilateral corneal opacity 22 cases $(3.66 \%)$

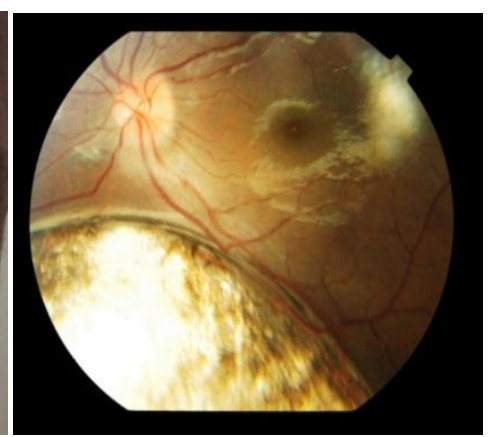

Chorioretinal Coloboma $2 \operatorname{cases}(0.33 \%)$

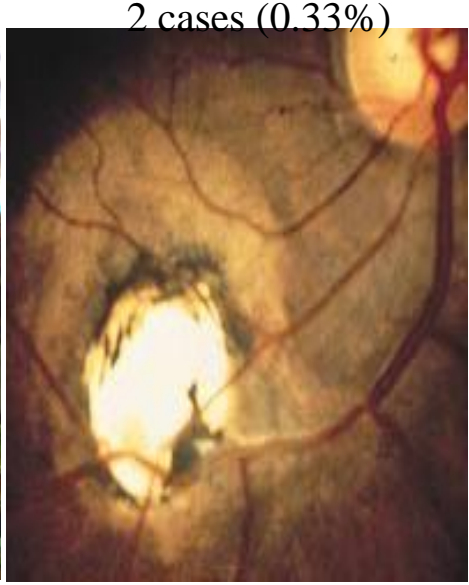

Toxoplasma

Chorioretinitis

Table 1 shows type of cataract and no. of cases

\begin{tabular}{|l|l|l|}
\hline Type of Cataract & No. of Cases & \% of Cases \\
\hline 1) Nuclear Cataract & 26 & $27.08 \%$ \\
\hline 2) Lamellar Cataract & 22 & $22.91 \%$ \\
\hline 3) Blue dot opacities & 8 & $8.33 \%$ \\
\hline 4) Total Congenital Cataract & 7 & $7.29 \%$ \\
\hline 5) Sutural Cataract & 3 & $3.12 \%$ \\
\hline 6) Coralliform Cataract & 3 & $3.12 \%$ \\
\hline 7) Floriform Cataract & 4 & $4.16 \%$ \\
\hline 8) Posterior Polar Cataract & 4 & $4.16 \%$ \\
\hline 9) Rubella Syndrome & 3 & $3.12 \%$ \\
\hline 10) Coronary Cataract & 2 & $2.08 \%$ \\
\hline 11) Anterior Polar Cataract & 1 & $1.04 \%$ \\
\hline 12) Punctate Subcapsular Cataract & 1 & $1.04 \%$ \\
\hline 13) Traumatic Cataract & 12 & $12.5 \%$ \\
\hline
\end{tabular}


Shows type of cataract
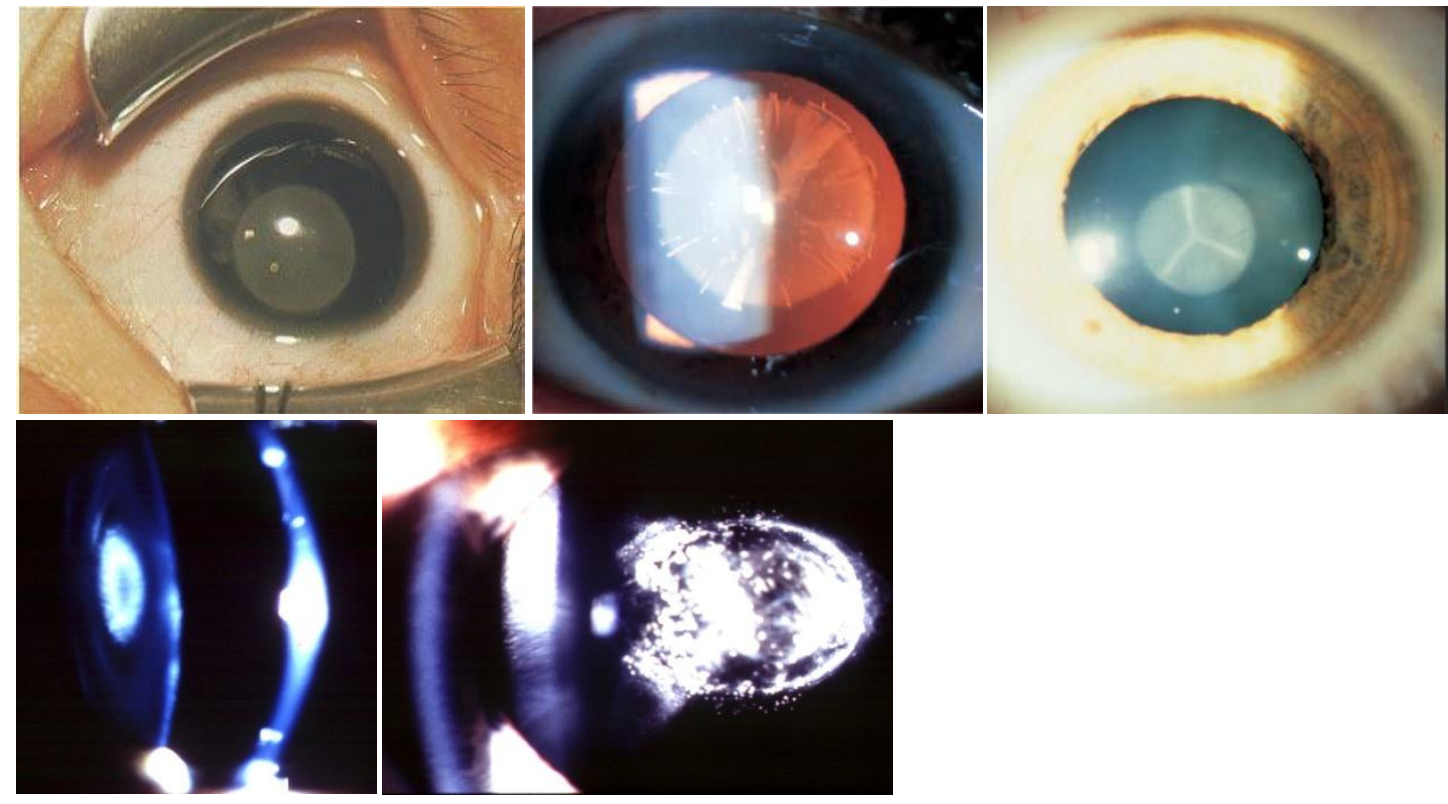

Central

Posterior Polar Cataract

Pulverulent

Cataract

In our study, 70 cases $(11.66 \%)$ of Ptosis were seen.

Table 2 shows no. of cases of Ptosis

\begin{tabular}{|l|l|l|}
\hline Grade of Ptosis & No. of Cases & \% of Cases \\
\hline 1) Mild Ptosis & 25 & $35.71 \%$ \\
\hline 2) Moderate Ptosis & 34 & $48.57 \%$ \\
\hline 3) Severe Ptosis & 11 & $15.71 \%$ \\
\hline
\end{tabular}

Figure shows patients with Ptosis

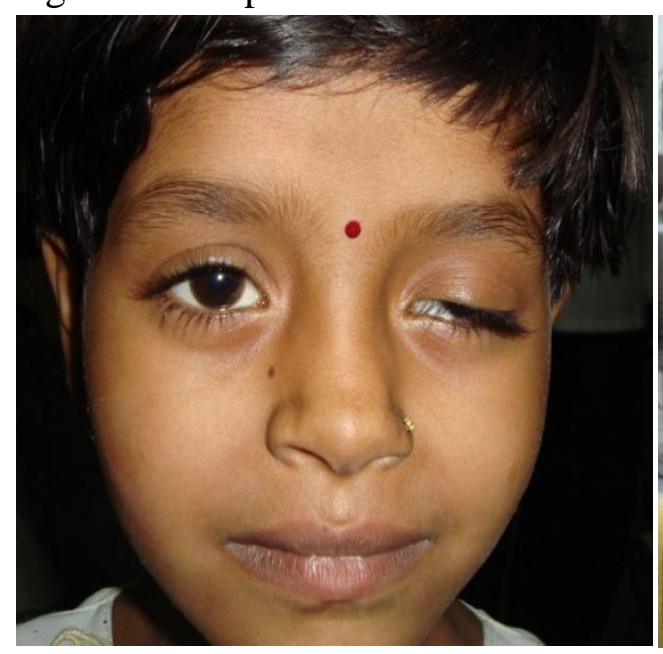

Severe Ptosis

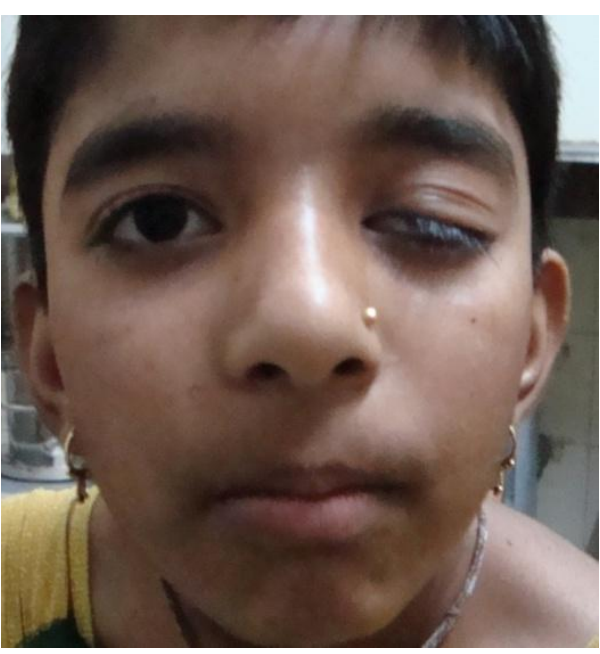

Severe Ptosis 
Patients of xerophthalmia presented with night blindness, conjunctival xerosis, bitot spots, bilateral punctate corneal epithelial erosions, sterile corneal melting by colliquative necrosis (keratomalacia).

Figures shows cases of Xerophthalmia

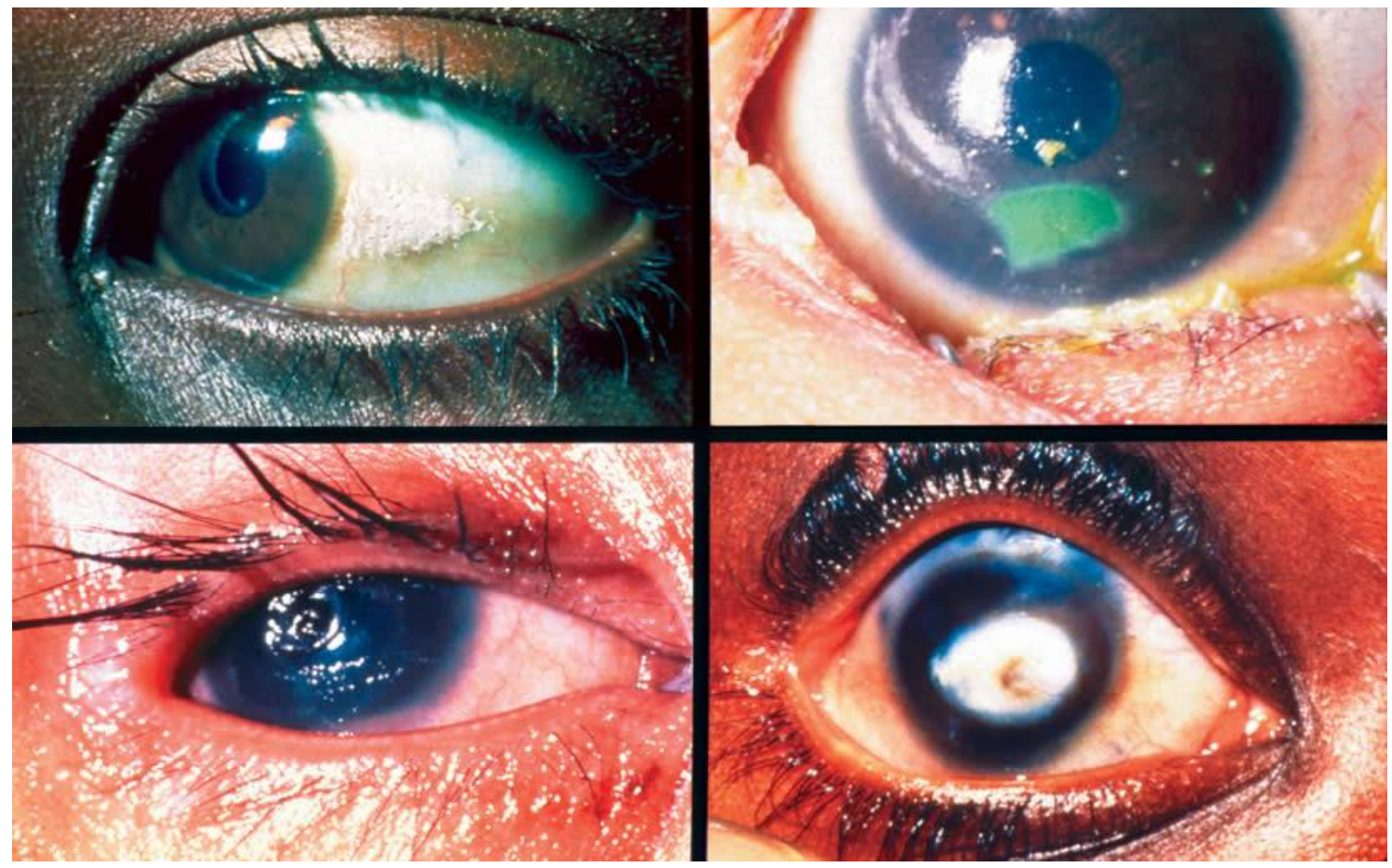

Xerophthalmia is caused by Vitamin A deficiency.

Treatment of Xerophthalmia :

Children over one year

- Immediately on diagnosis (Day 1): 200,000 IU vitamin A orally ${ }^{+}$

- The following day (Day 2): 200,000 IU vitamin A orally

- Four weeks later (Week 4): 200,000 IU vitamin A orally

+ If there is vomiting, an intramuscular injection of $100,000 \mathrm{IU}$ of water soluble vitamin A (not an oil-based preparation) may be used instead of the first oral dose.

Children under one year old or $<8 \mathrm{~kg}$

- Use half the doses of the regimen given above. 
Retinoblastoma is the most common primary intraocular malignancy of childhood. In our study, 2 cases $(0.33 \%)$ of retinoblastoma were seen.

Figures shows Retinoblastoma

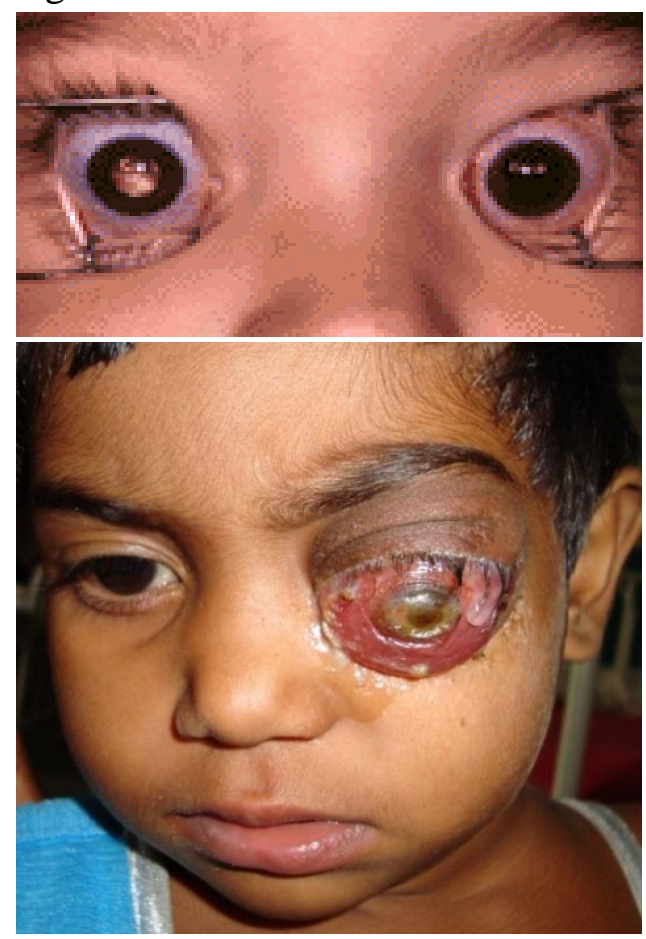

Presentation is within first year of life in bilateral cases and around 2 years of age if the tumour is unilateral.

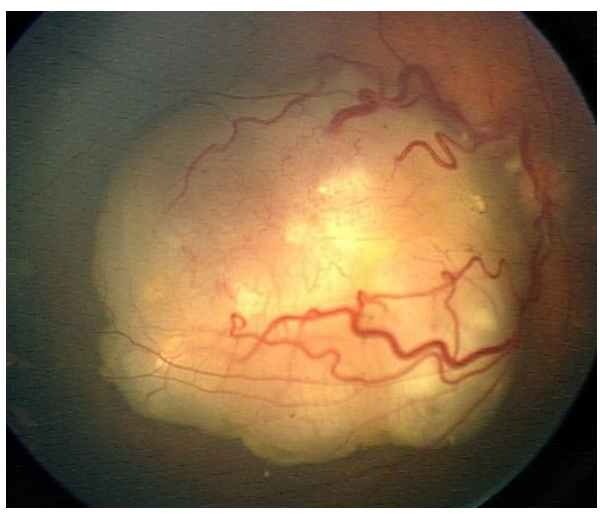

Retinoblastoma presented with leukocoria, strabismus, secondary glaucoma, orbital inflammation.

Treatment of small tumours:

1. Photocoagulation using $532 \mathrm{~nm}$ argon or 810nm diode laser.

2. Cryotherapy

3. Chemotherapy

Treatment of medium sized tumours:

1. Brachytherapy using Iodine- 125 or Ruthenium-106.

2. Primary chemotherapy with intravenous carboplatin, etoposide and vincristine (CEV).

Treatment of large tumours:

1. Chemotherapy to shrink the tumour.

2. Enucleation is indicated if there is rubeosis, vitreous haemorrhage or optic nerve invasion. It is imperative to obtain a long piece of optic nerve (12-15mm).
In our study, $15.33 \%$ (92 cases) of visual impairment was due to uncorrected refractive error.

Globally refractive error is one of the most common cause of visual impairment and second leading cause of treatable blindness.

School children constitute a particular vulnerable group and uncorrected refractive errors can have detrimental effect on the academic, social and later the functional potential of individuals.

Screening programmes which incorporate the provision of glasses for children with significant refractive error are simple and effective means of eliminating this problem.

In our study, 80 cases $(13.33 \%)$ of Amblyopia were seen. 
Amblyopia is the unilateral or rarely bilateral decrease of best corrected visual acuity caused by form vision deprivation and/or abnormal binocular interaction, for which there is no pathology of the eye or visual pathways.

Strabismic Amblyopia: Results from abnormal binocular interaction where there is continued monocular suppression of the deviating eye.

Stimulus Deprivation Amblyopia: Caused due to form vision deprivation of the eye due to media opacity such as cataract.

Anisometropic Amblyopia : It is caused by a difference in refractive error. It results from abnormal binocular interaction from the superimposition of focused and unfocused images.

\section{Management:}

Eliminating any obstacle to vision such as a cataract.Correcting refractive error by full cycloplegic refraction.

Occlusion of the normal eye to encourage use of the amblyopic eye is the most effective treatment.Penalization in which vision in normal eye is blurred with atropine is an alternative method.

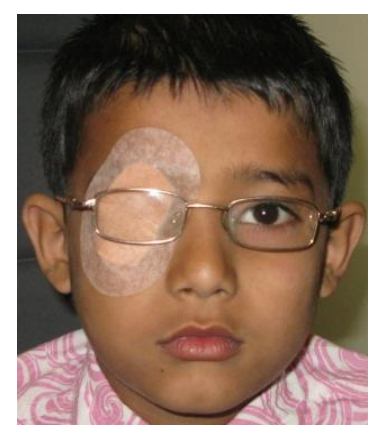

\section{DISCUSSION}

Childhood blindness refers to a group of diseases and conditions occurring in childhood, which, if left untreated, result in blindness or severe visual impairment that are likely to be untreatable later in life.Due to enormous loss of Disability Adjusted Life Years (DALYs), childhood blindness is estimated to be the second leading cause of the burden due to blindness. Visual disabilities in children are more complex compared to those in adults. Without visual stimulus, the child's overall development suffers. Because of the country's size, the eye care sector in India faces several major challenges including lack of access for rural populations, unequal distribution of services, gross underutilization of available services, and insufficient facilities. Various international and national government aided project have been started to reduce the load of childhood blindness. This study was carried out to know various causes of visual disabilities and its management at AVBRH a rural setup.

In the present study, of the 600 children studied, we found the cases to be as Cataract (congenital, developmental, traumatic) $16 \%$, Refractive error (myopia, hypermetropia, astigmatism) 15.33\% , Congenital globe anomalies (microphthalmos, anophthalmos) $4.99 \%$, Ptosis $11.6 \%$, Corneal disorders (opacity, keratoconus) $10.48 \%$, Posterior segment pathologies (macular dystrophy, ROP, Retinoblastoma, Coat's disease, Toxoplasma chorioretinitis, Pathological myopia, Traumatic R.D) $7.29 \%$, Amblyopia $13.33 \%$, Ocular Trauma $3 \%$, Strabismus $25.5 \%$, Idiopathic Nystagmus $3.66 \%$, Xerophthalmia 3\%.

Lalit Dandona, Clare E.Gilbert, Jugnoo S. Rahi, Gullapali N. Rao in 1998 showed planning to reduce childhood blindness in India $^{1}$. The population -based estimation of childhood blindness reported for rural population of West Godava Dist.,in Andra Pradesh. In 1,13,514 children the prevalence of blindness was found to be 6.5 per 10,000 children. Main causes were congenital globe anomalies (microphthalmos /anophthalmos) in $25 \%$, retinal diseases $22.2 \%$, optic atrophy $16.7 \%$, cataract $15.3 \%$, corneal opacity $11.1 \%$, Vit.A deficiency $5.5 \%$, glaucoma $4.2 \%$, Amblyopia $2.8 \%$, abnormalities of visual pathway $2.8 \%$. Incidence of corneal opacity and cataract is comparable with our present study.

Dandona L, Rahi JS et al in another study of 1318 blind school students in 9 states of India revealed 
that the major causes were corneal scarring due to Vit. A deficiency in $26.4 \%$, globe anomalies in $20.7 \%$, retinal diseases in $19.3 \%$, cataract $12.3 \%$ \& optic nerve disorders in $5.9 \%$.

Childhood blindness is one of the priorities in Vision 2020: the right to sight $^{2}$. Causes of blindness in children vary according to region and socioeconomic development ${ }^{3,4}$. In the context of Vision 2020, the priorities for action to reduce childhood blindness in India are refractive error, cataract related amblyopia, and corneal diseases.Blindness in childhood may interfere with the normal development and education of a child.Approximately one-third of the total economic cost of blindness is thought to be due to childhood blindness.

In the present study, $15.33 \%$ of visual impairment was due to uncorrected refractive error.

Globally refractive error is one of the most common causes of visual impairment and second leading cause of treatable blindness ${ }^{6}$.

Schoolchildren constitute a particular vulnerable group, and uncorrected refractive errors can have detrimental effect on the academic, social and later the functional potential of individuals.

The prevalence of refractive errors in school children ranges from $1 \%$ to $23.3 \%^{7,8}$.About 60 $80 \%$ of visual impairment may be due to refractive error alone.

In the present study, of the 600 patients , 260 (43.33\%) were found to have bilateral blindness or subnormal vision and 340 (56.66\%) were found to have unilateral blindness or subnormal vision. The causes for bilateral blindness and subnormal vision included cataract, microphthlmos, Vit. A deficiency, refractive error, macular dystrophy, idiopathic nystagmus, albinism. The causes for unilateral blindness and subnormal vision included amblyopia, ocular trauma, cataract , ptosis , corneal opacity.

It was observed by Yorston D in 1999 that worldwide major causes of blindness in children include retinal dystrophies, congenital cataract, congenital glaucoma, corneal opacities, trauma related complications, optic atrophy and vitamin A deficiency. ${ }^{9}$

In the present study, of the whole globe anomalies, $6(1 \%)$ cases were phthisis bulbi, 4 $(0.66 \%)$ cases were anophthalmos, 15 (2.5\%) cases were microphthalmos, $5(0.83 \%)$ were congenital glaucoma.

Complex (colobomatous ) microphthalmos is associated with coloboma, usually of the iris.

Patients with congenital glaucoma present with lacrimation, photophobia, blepharospasm and corneal haze. Investigations done for patients with congenital glaucoma were measurement of IOT and corneal diameter, gonioscopy. Treatment for patients with congenital glaucoma included goniotomy, trabeculotomy and trabeculectomy. Patients were followed up after surgery and IOT and corneal diameters were monitored at regular intervals.

Unfortunately, the parents of many children with congenital glaucoma delay seeking medical advice until the disease has reached the end stage.

In the present study, corneal opacity was found in $29(4.83 \%)$ cases, of which 4 were bilateral and 25 were unilateral.

In the present study, penetrating keratoplasty was done in 4 patients and optical iridectomy was done in 5 patients.

Causes of corneal opacities in children were healed keratomalacia, healed keratitis , traumatic corneal opacity, measles, Ophthalmia neonatorum, harmful traditional eye medicines. Penetrating keratoplasty has an extremely high risk for failure in children, with a variable visual and anatomic outcome. $^{10,11 .}$ Optical iridectomy is a surgical alternative to penetrating keratoplasty. Optical iridectomy creates a clear entrance pupil, improving vision in patients with segmental corneal opacities. An area of clear peripheral cornea can produce retinal images compatible with good visual acuity. Optical iridectomy aims to provide ambulatory vision in the patient who, if 
left unoperated, would end up being deeply amblyopic. The timing of intervention is critical to prevent the development of irreversible, dense amblyopia.

In the present study, 25cases (4.16\%) of Vitamin A deficiency were seen.

In a study in 2001, Lalit Dandona et $\mathrm{al}^{5}$ observed $5.5 \%$ of cases of Vitamin A deficiency, which is comparable to our present study.

Xerophthalmia is caused by Vitamin A deficiency. Patients of xerophthalmia present with night blindness, conjunctival xerosis, bitot spots, bilateral punctuate corneal epithelial erosions, sterile corneal melting by colliquative necrosis (keratomalacia). Oral or Intramuscular Vit. A was provided as treatment. In children over one year of age, immediately on diagnosis (Day 1), 200000 IU Vit.A was given orally. On day 2, 200000 IU Vit.A was given orally. Four weeks later (Week 4), 200000 IU Vit.A was given orally. In children under one year old or $<8 \mathrm{kgs}$, half the doses of the above regimen were given. Intake of dietary sources of Vit.A was encouraged.

According to World Development Report published in Oxford University Press ${ }^{12}$ in 1993, the most common cause of visual loss in children in developing countries is vitamin A deficiency, which accounts for up to $70 \%$ of cases in some countries. WHO estimates that 13.8 million children worldwide have some degree of visual loss attributable to vitamin A deficiency.

In the present study, $96(16 \%)$ cases of cataract were seen, of which 64 were bilateral and 32 were unilateral. $62(64.58 \%)$ cases were of congenital cataract, $22(22.91 \%)$ of developmental cataract and $12(12.5 \%)$ traumatic cataract.

In the present study, $26(27.08 \%)$ cases were nuclear cataract, $22(22.91 \%)$ were lamellar type, $8(8.33 \%)$ were blue dot opacities, 7 (7.29\%) were total congenital cataract, $3(3.12 \%)$ were sutural type , $3(3.12 \%)$ were coralliform cataract, 4 $(4.16 \%)$ were floriform cataract, 4 (4.16\%) posterior polar cataract, $3(3.12 \%)$ congenital rubella syndrome, 2 (2.08\%) were coronary type, $1(1.04 \%)$ anterior polar cataract associated with aniridia, $1(1.04 \%)$ punctate subcapsular cataract and $12(12.5 \%)$ traumatic cataract.

In the present study, investigations done in a case of congenital cataract included Toxoplasmosis titre, Rubella titre, Cytomegalovirus titre, Herpes Simplex titre (Serology for intrauterine infection), Urine analysis for reducing substance after drinking milk (galactosaemia), Chromatography for amino acids (Lowe syndrome), Sr. Calcium, Sr. Phosphorus, Red blood cell GPUT, galactokinase levels, CBC, RBS, B-Scan, USG using Doppler- to diagnose persistent anterior fetal vasculature since blood flow may be seen in a persistent hyaloid artery, VEP wherever required, Paediatrician reference to rule out systemic disorders.

In the present study, surgical management of cataract cases included $\mathrm{ECCE}+\mathrm{PCC}+\mathrm{AV}+\mathrm{PCIOL}$ in $37(38.54 \%)$ children, $\mathrm{ECCE}+\mathrm{PCC}+\mathrm{PCIOL}$ in $21(21.87 \%)$ children , $27(28.12 \%)$ underwent ECCE+PCIOL , $6(6.25 \%)$ underwent ECCE , 4 $(4.16 \%)$ underwent ECCE+PCC+AV , 1(1.04\%) underwent ICCE.

Surgery involved anterior capsulorrhexis, aspiration of lens matter, capsulorrhexis of the posterior capsule, limited anterior vitrectomy and IOL implantation.

In the present study, pre-operative visual acuity of children having cataract ranged from $\mathrm{PL}+$ to CF 6mts. After cataract surgery, 43(44.79\%) patients showed post-operative visual acuity of $6 / 9$ to $6 / 18,38(39.58 \%)$ patients showed postoperative visual acuity of $6 / 24,10(10.41 \%)$ patients had post-operative visual acuity of $6 / 36$, and $5(5.20 \%)$ patients had post-operative visual acuity of CF 6mts to $6 / 60$.

\section{Post-operative Complications:}

1) PCO: Posterior capsule opacification is universal if posterior capsule is retained in child under the age of 6 years. 
a. There is opacification of anterior hyloid face despite posterior capsulorrhexis if anterior vitreous is left intact.

b. Incidence of opacification is reduced when posterior capsulorrhexis is combined with vitrectomy.

2) Secondary membranes associated with chronic uveitis.

3) Proliferation of lens epithelium leads to formation of Soemmering ring. As it is located peripherally, it is visually inconsequential.

4) Closed angle glaucoma can develop in microophthalmic eyes secondary to pupil block.

\section{Visual Rehabilitation:}

1) IOL implantation: Awareness of the rate of myopic shift which occurs in the developing eye, combined with accurate biometry, allowed the calculation of an IOL power targeted at initial hypermetropia (correctable with spectacles) which ideally decay towards emmetropia later in life.

2) Spectacles - for older children with bilateral aphakia.

3) Contact lenses - superior optical solution for both unilateral and bilateral aphakia.

Trauma is the most common cause of subluxation of crystalline lens. Common causes of spontaneous subluxation include Marfan's syndrome, Weil-Marchesani syndrome, and homocystinuria.

In the present study, $4(0.66 \%)$ cases of macular dystrophy were seen, having bilateral involvement.

Stargardt disease is the most common form of juvenile-onset macular dystrophy. There is bilateral, gradual impairment of central vision. Fundus showed oval, 'snail-slime' or 'beatenbronze' foveal appearance, which may be surrounded by yellow-white flecks. Some cases showed geographic atrophy with bull's eye configuration. Prognosis is poor and once visual acuity drops below 6/12, it tends to decrease rapidly and stabilize at about $6 / 60$.

In the present study, $3(0.5 \%)$ cases of retinopathy of prematurity (ROP) were seen.

Babies born at or before 31 weeks gestational age, or weighing $1500 \mathrm{gm}$ or less, should be screened for ROP.

ROP shows following stages:

Stage 1- Demarcation line

Stage 2- Ridge

Stage 3 - Extra retinal fibrovascular proliferation

Stage 4 - Partial Retinal Detachment

Stage 5- Total Retinal Detachment

$80 \%$ cases of ROP regress spontaneously by a process of involution or evolution from a vasoproliferative to fibrotic phase. Laser photocoagulation of avascular immature retina is recommended in infants with threshold disease. Lens- sparing pars plana vitrectomy is performed for tractional retinal detachment not involving the macula.

In the present study, $2(0.33 \%)$ cases of retinoblastoma were seen.

Retinoblastoma is the most common primary intraocular malignancy of childhood. Presentation is within the first year of life in bilateral cases and around 2 years of age if the tumour is unilateral. ${ }^{13}$. Retinoblastoma can present with leukocoria, strabismus, secondary glaucoma, orbital inflammation.

Indirect ophthalmoscopy with scleral indentation was performed on both eyes after full mydriasis. Investigations done in retinoblastoma include:

B-Scan USG- to detect calcification

CT Scan - to detect calcification

MRI- for optic nerve evaluation. 


\section{Treatment of small tumours:}

1) Photocoagulation using $532 \mathrm{~nm}$ argon or 810nm diode laser

2) Cryotherapy

3) Chemotherapy

\section{Treatment of medium-sized tumours:}

1) Brachytherapy using Iodine-125 or Ruthenium-106.

2) Primary chemotherapy with intravenous carboplatin, etoposide and vincristine (CEV).

\section{Treatment of large tumours:}

1) Chemotherapy to shrink the tumour.

2) Enucleation is indicated if there is rubeosis, vitreous haemorrhage or optic nerve invasion. It is imperative to obtain a long piece of optic nerve ( $12-15 \mathrm{~mm})$.

In the present study, $5(0.83 \%)$ cases of pathologicalmyopia were seen.

High myopia is defined as an eye with a refractive error $>-6 \mathrm{D}$ and an axial length of the globe $>26 \mathrm{~mm}$. Pathological or degenerative myopia is characterized by progressive and excessive anteroposterior elongation of the globe, which is associated with secondary changes involving the sclera, retina, choroid and optic nerve head.

Myopic retinopathy includes the presence of staphyloma, lacquer cracks, Fuchs' spot, myopic chorioretinal thinning or atrophy, peripapillary atrophy, cytotorsion or tilting of the optic disc, and the $\mathrm{T}$ sign found in central retinal vessels. Maculopathy is the most common cause of visual loss in highly myopic patients. ' Lacquer cracks' consists of ruptures in the RPE-Bruch membranechoriocapillaries complex characterized by fine, irregular, yellow lines, often branching and crisscrossing at the posterior pole. Foster-Fuchs spot is a raised, circular, pigmented lesion that may develop after a macular haemorrhage has absorbed. Choroidal neovascularisation is one of the most common causes of vision loss in pathological myopia. ${ }^{14}$.

\section{Complications:}

1) Staphylomas- May be associated with macular hole formation.

2) Rhegmatogenous retinal detachment

3) Foveal retinoschisis

In the present study, $2(0.33 \%)$ cases of traumaticretinaldetachment were seen.

Trauma is responsible for about $10 \%$ of all cases of R.D and is the most common cause in children, particularly boys. ${ }^{15}$. Penetrating injuries of the posterior segment carry a high risk of R.D, particularly if there is vitreous incarceration at the site of penetration, which subsequently leads to vitreoretinal traction. Severe blunt trauma causes a compression of the anteroposterior diameter of the globe and a simultaneous expansion at the equatorial plane.

Rhegmatogenous retinal detachments in children are often found accompanying other vitreoretinal pathology or trauma and has been reported in cases of Marfan's syndrome, Stickler's syndrome, cataract extraction, myopia. Less frequent causes are Toxocara, Coat's disease, retinopathy of prematurity (ROP), persistent hyperplastic primary vitreous (PHPV) and Eales' disease. ${ }^{16 .}$

The most frequent management procedures for R.D are pars-plana vitrectomy (PPV), intraocular gas (SF6), external band with or without internal drainage, and cryotherapy or laser.

\section{Management of R.D includes:}

- $\mathrm{PPV}^{*}+$ silicone oil \pm internal drainage \pm External band \pm cryotherapy/ laser

- $\quad \mathrm{PPV}+$ Intra- ocular Gas + External band \pm Internal drainage + cryotherapy/ laser

- External band \pm external drainage PPV + Lensectomy \pm External band+ laser \pm internal drainage \pm Silicone oil

- $\mathrm{PPV}+$ Removal of IOFB**

*Pars- plana vitrectomy.

**Intra- Ocular foreign body. 
In the present study, $80(13.33 \%)$ cases of Amblyopia were seen.

Amblyopia is the unilateral or rarely bilateral decrease of best- corrected visual acuity caused by form vision deprivation and/or abnormal binocular interaction, for which there is no pathology of the eye or visual pathways.

\section{Amblyopia can be:}

1) Strabismic amblyopia

2) Anisometropic amblyopia

3) Stimulus deprivation amblyopia

4) Bilateral ametropic amblyopia

5) Meridional amblyopia

In the absence of an organic lesion, a difference in best corrected visual acuity of two Snellen lines or more is indicative of amblyopia.

The sensitive period during which acuity of an amblyopic eye can be improved is usually upto 7 -8years in strabismic amblyopia. Occlusion of the normal eye, to encourage use of the amblyopic eye is the most effective treatment. Penalization in which vision in normal eye is blurred with atropine is an alternative method.

Three critical periods of human visual acuity development have been determined. ${ }^{17}$. During these time periods, vision can be affected by the various mechanisms to cause or reverse amblyopia. These periods are as follows:

- The development of visual acuity from the $6 / 60$ range to $6 / 6$, which occurs from birth to age 3-5 years.

- The period of the highest risk of deprivation amblyopia, from a few months to 7 or 8 years.

- The period during which recovery from amblyopia can be obtained, from the time of deprivation up to the teenage years or even sometimes the adult years.

The Amblyopia Treatment Studies have helped to define the role of full-time patching versus parttime patching in patients with amblyopia. The studies have demonstrated that, in patients aged 3-
7 years with severe amblyopia (visual acuity between 20/100 and 20/400), full-time patching produced a similar effect to that of 6 hours of patching per day. In a separate study, 2 hours of daily patching produced an improvement in visual acuity similar to that of 6 hours of daily patching when treating moderate amblyopia (visual acuity better than 20/100) in children aged 3-7 years. Patching was prescribed in combination with 1 hour of near visual activities.

Data from the Amblyopia Treatment Studies are also available for older patients. For patients aged from 7 years to younger than 13 years, the Amblyopia Treatment Studies have suggested that prescribing 2-6 hours a day of patching can improve visual acuity even if the amblyopia has been previously treated. For patients aged from 13 years to younger than 18 years, prescribing 2-6 hours a day of patching might improve visual acuity when amblyopia has not been previously treated; however, this is likely to be of little benefit if amblyopia was previously treated with patching.

In the present study, $70(11.66 \%)$ cases of ptosis were seen, of which 63 were unilateral and 7 were bilateral.

Of the 70 cases, 25 cases were mild ptosis, 34 were moderate ptosis and 11 were severe ptosis.

In the present study, ptosis correction was done by Fasanella-Servat surgery in 19 patients, LPS Resection in 40 patients and Frontalis Suspension in 11 patients.

\section{CONCLUSION}

Preventable causes of blindness (Xerophthalmia) can be tackled by improving the primary levels of health care delivery (Vitamin A Supplementation, Rubella \& Measles Immunisation).

Treatable causes (Cataract, Refractive Errors) require system for early identification and referral and increased public awareness.

Early detection is important as it greatly improves the ultimate visual prognosis of children 
with congenital cataract, uncorrected refractive error, amblyopia, congenital glaucoma.

Creating publicawareness, screening programmes in community and school, cooperation of parents are necessary for management of ocular diseases in children.

Prevention and management of childhood blindness is one of the priorities of Vision 2020: The Right to Sight.

Intiative taken and work done by programmes like Sarv Shiksha Abhiyan ( Govt. of India's flagship programme) and ORBIS ( an international nonprofit NGO) for prevention and control of childhood blindness is appreciable.

\section{BIBLIOGRAPHY}

1. Lalit Dandona, Clare Gilbert, Jugnoo Rahi, Gullapalli Rao. Planning to reduce childhood blindness in India. I.J.O 1998: 46 ; 117-122.

2. World Health Organization. Global initiative for the elimination of avoidable blindness. Programme for the Prevention of Blindness and Deafness. Geneva: WHO, 1997 (WHO/PBL/97.61).

3. World Health Organization. Preventing blindness in children: report of WHO/IAPB scientific meeting. Programme for the Prevention of Blindness and Deafness, and International Agency for Prevention of Blindness. Geneva: WHO, 2000 (WHO/PBL/00.77).

4. Gilbert CE, Anderton L, Dandona L, et al. Prevalence of visual impairment in children: a review of the available data. Ophthalmic Epidemiol 1999;6:73-82.

5. Dandona L, Dandona R, Srinivas M, et al. Blindness in the Indian state of Andhra Pradesh. Invest Ophthalmol Vis Sci 2001;42:908-16.

6. Dandona R \& Dandona L. refractive error blindness. Bulletin of the World Health Organisation. 2001; 79: 237-243.
7. Murthy GVS, Gupta S, Ellwein LB, et al. refractive error in children in an urban population in New Delhi. Investigative Opthalmology \& Visual Science. 2002; 43: 623-631.

8. Kalikivayi V, Naduvilath TJ, Bansal AK, Dandona L. Visual impairment in school children in Southern India. Indian J Ophthalmology. 1997; 45:129-134.

9. Yorston D. The global initiative Vision 2020: the right to sight. Childhood blindness. Community eye health, 1999, 12(31):44-5.

10. Aasuri MK, Garg P, Gokhle N, Gupta S. Penetrating keratoplasty inchildren. Cornea 2000;19:140-4.

11. McClellan K, Lai T, Grigg J, Billson F. Penetrating keratoplasty inchildren: visual and graft outcome. $\mathrm{Br} \mathrm{J}$ Ophthalmol 2003;87:1212-4.

12. World Bank. Investing in health. World development report.Oxford: Oxford University Press, 1993.

13. Hungerford J. Factors influencing metastasis in retinoblastoma. $\mathrm{Br} J$ Ophthalmol. Sep 1993;77(9):541.

14. Virgili G, Menchini F, Virgili G. Laser photocoagulation for choroidal neovascularisation in pathologic myopia. Cochrane Database Syst Rev 2005. $19 C D 004765$.

15. Sarrazin L, Averbukh E, Halpert M, Hemo I, Rumelt S. Traumatic paediatric retinal detachment: A comparison between Open and closed globe injuries. Am J Ophthalmol 2004; 137: 1042- 1049.

16. Fivgas GD, Capone A Jr. Paediatric rhegmatogenous retinal detachment. Retina. 2001;21 (2):101-6.

17. Daw NW. Critical periods and amblyopia. Arch Ophthalmol. Apr 1998;116 (4):502-5. 